concerned, a large firm, with the assistance of the relevant government services, would be at least as safe as the present organization. $\mathrm{He}$ thinks that security troubles have come rather from failures in detection than from not being ruthless enough in acting on evidence less than legal proof.

Prof. H. S. W. Massey concurs in these views. Most physicists think that Harwell has succeeded to a remarkable extent, but that development would best be carried out by a body of the corporation type retaining the maximum possible freedom and initiative. It is a very different matter, however, to change an established system which is working well, and Prof. Massey thinks that suggestions that a corporation could tighten up security by decreasing security of tenure or restricting civil rights, such as travel, are particularly ill-advised.

A statement made by Prof. M. H. I. Pryce represents the views of scientific men directly concerned with atomic energy work. There appears to be general agreement in the atomic energy establishments that certain features of the present organization, such as the rigid staffing system, are frustrating and should be changed; but a strong body of opinion holds that the situation can be remedied within the Civil Service by giving greater autonomy to the various establishments. This view is particularly strong in the production establishments, whereas those more closely concerned with the research side tend to share the general outside view of the unalterable inflexibility of Civil Service organization. The popular view that the Civil Service is inefficient is not strongly held at Harwell, and it was forcibly put to Prof. Pryce that this view is ill-informed and made by those without experience of the essential structure of large organizations. Prof. Pryce, moreover, makes an important point about the publicity policy of the Ministry of Supply. Few people know of the real achievements at Harwell, of which many sections of the Establishment should justifiably feel proud. Not only is there, accordingly, no feeling of pride in the Establishment, but also potential recruits into the atomic energy field are not attracted ; and the belief, fostered by the Ministry's attitude, that most of the work at Harwell is secret, deters others from joining. Opinion, however, in the establishments appears to be crystallizing in favour of remaining in the Civil Service, and this tendency has been strengthened by Lord Swinton's comments in the House of Lords on security conditions and the possible loss of civil rights and fears of arbitrary dismissal under a new system.

\section{COMETS AND THEIR ORIGIN}

$\mathrm{T}$ HE presidential address to the British Astronomical Association ${ }^{1}$, delivered by Dr. G. Merton on October 31, was on the subject of "Comets and their Origin". Dr. Merton gave a short historical survey of the subject, followed by a description of the different parts of these bodies, their composition, their appearances more particularly as they approach perihelion, the diversity in their orbits and the peculiarities exhibited by certain comets regarding sudden increases in brightness, etc., and then he proceeded to his main thesis on the origin of comets. Before considering some of the older theories, he referred to two modern ones which depend upon the passage of the sun through a cloud of interstellar dust, or upon the debris of an exploded planet, this debris-or a very small fraction of it-afterwards becoming a cloud of comets. Neither theory is really new, but important details have been worked out within the past few years and differ from many of those previously suggested; there are differences in other ways as well. The first of these, by $R$. A. Lyttleton, has already been mentioned in Nature $^{2}$, and so in this article it is only necessary to refer to the other that appeared a little later, namely, that due to J. H. Oort ${ }^{3}$.

Oort's theory postulates the explosion of a planet between the orbits of Mars and Jupiter, the fragments with approximately circular orbits around the sun becoming minor planets and meteors. Portions with elliptical orbits which approached Jupiter or other planets were subjected to perturbations which, taken on the whole, increased the major axes of their orbits, and by far the greater part of the debris, thrown into hyperbolic orbits, was lost to the solar system. About 3 per cent of the debris moved in elliptical orbits with very large major axes-from about 25,000 to 200,000 astronomical units-and this portion formed the outer clouds of comets which have supplied the solar system with these bodies since the explosion took place, and will continue to do so for a long time, as the estimated number of comets is of the order of two hundred thousand million. On the assumption that the exploded planet had a mass about that of the earth and also that 3 per cent of it formed the outer cloud, the average mass of a comet would be about ten thousand million tons; but the above figures merely denote the order of number and mass.

Assuming that the cloud had a random distribution of directions with respect to the sun, a small proportion of the comets would cross a sphere of radius about two astronomical units, the sun being at its centre; and van Woerkom, an associate of Oort at Leyden Observatory, has shown that such comets would be forced into hyperbolic orbits and ejected from the solar system or converted into short-period comets. Perturbations by the stars are responsible for supplying new comets to this small inner sphere.

The details of the theory are very fully dealt with in Oort's original paper, but in the limited space allotted for Dr. Merton's address it was impossible to explain all these. (It may be remarked that the explosion of a planet with the subsequent production of minor planets and meteorites have been dealt with by W. H. Ramsey and M. J. Lighthill in some papers which appeared in the Monthly Notices of the Royal Astronomical Society, references to which have been made in Natures, though it is admitted that the complexity of the problem prevents a complete quantitative treatment.) Towards the end of his address Dr. Merton referred to some of the theories of the origin of the periodic comets : the capture theory, which postulates a close approach of longperiod comets to some of the giant planets the perturbations of which turned them into relatively short-period comets; and the theory that they were ejected by the planets or even by the sun. As he pointed out, the great difficulty about all ejection theories is the absence of any positive evidence to support them, especially as the processes of ejection are supposed to be still going on.

${ }^{1}$ J. Brit. Astro. Assoc., 62, 1 (1951).

2 Nature, 164, 119 (1950).

8 Bull Astro. Inst. Netherlands 11 No 408 (1950); an ahbreviated account appeared in Sky and Telescope, 9, No. 4 (1950).

4 Nature, 163, 814 (1940); 165, 217 (1950); 167, 936 (1951); and 168, $676(1951)$. 